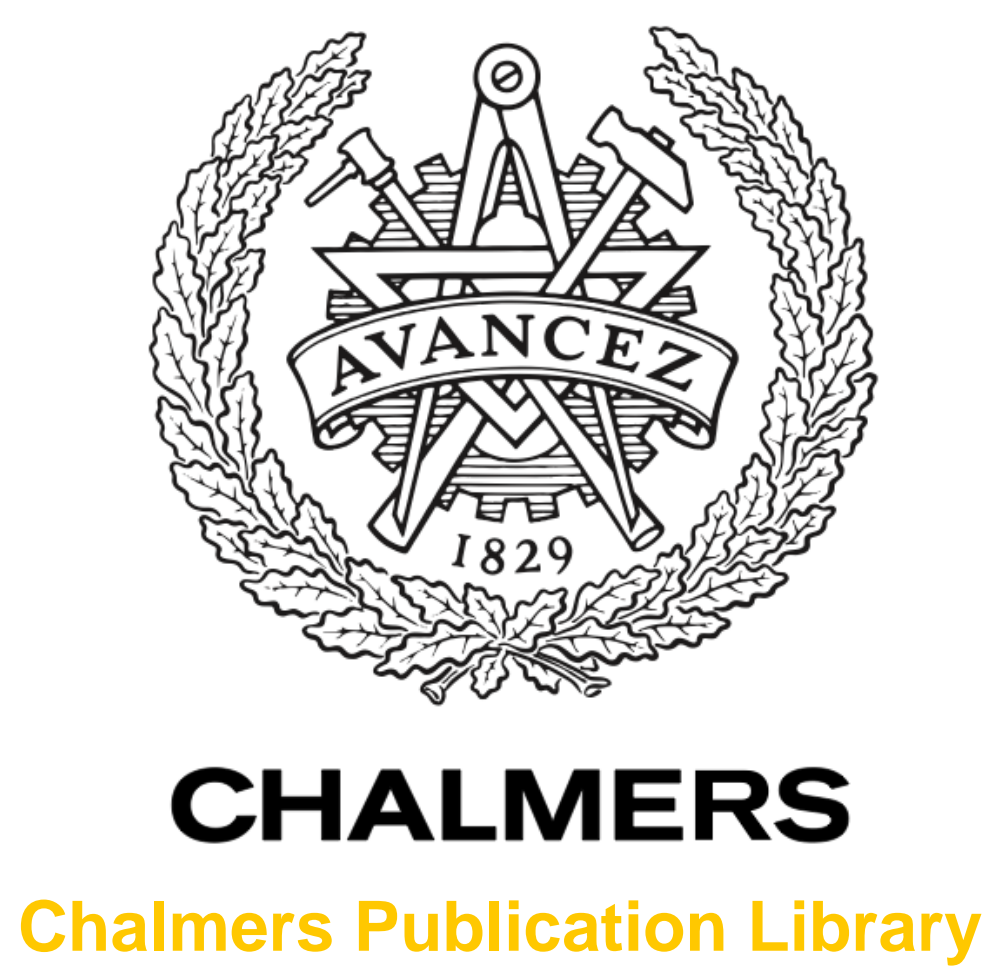

\title{
Thermal creation of electron spin polarization in n-type silicon
}

This document has been downloaded from Chalmers Publication Library (CPL). It is the author's version of a work that was accepted for publication in:

\section{Applied Physics Letters (ISSN: 0003-6951)}

Citation for the published paper:

Dankert, A. ; Dash, S. (2013) "Thermal creation of electron spin polarization in n-type

silicon". Applied Physics Letters, vol. 103(24), pp. article nr. 242405.

http://dx.doi.org/10.1063/1.4845295

Downloaded from: http://publications.lib.chalmers.se/publication/190527

Notice: Changes introduced as a result of publishing processes such as copy-editing and formatting may not be reflected in this document. For a definitive version of this work, please refer to the published source. Please note that access to the published version might require a subscription. 


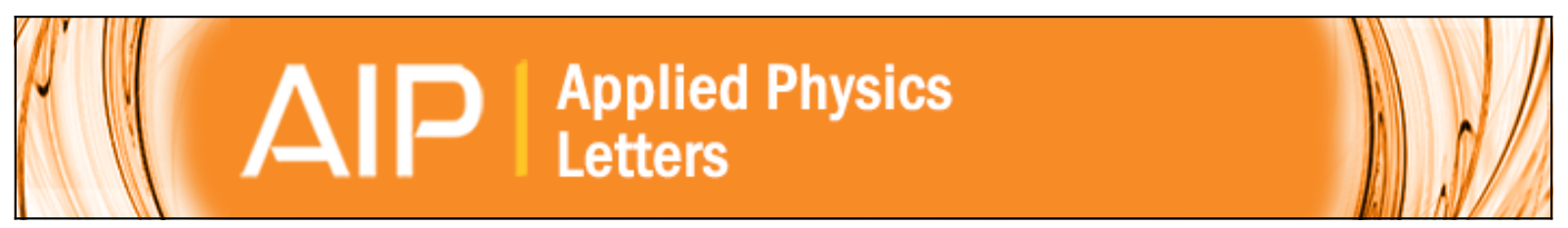

\section{Thermal creation of electron spin polarization in n-type silicon}

André Dankert and Saroj P. Dash

Citation: Applied Physics Letters 103, 242405 (2013); doi: 10.1063/1.4845295

View online: http://dx.doi.org/10.1063/1.4845295

View Table of Contents: http://scitation.aip.org/content/aip/journal/apl/103/24?ver=pdfcov

Published by the AIP Publishing

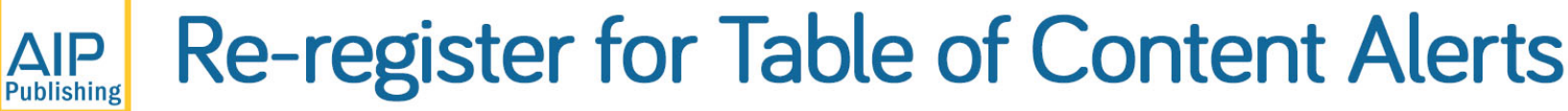

Create a profile.

Sign up today! 


\title{
Thermal creation of electron spin polarization in $\mathbf{n}$-type silicon
}

\author{
André Dankert ${ }^{\text {a) }}$ and Saroj P. Dash ${ }^{\text {b) }}$ \\ Department of Microtechnology and Nanoscience, Chalmers University of Technology, SE-41296 Göteborg, \\ Sweden
}

(Received 26 July 2013; accepted 23 November 2013; published online 10 December 2013)

\begin{abstract}
Conversion of heat into a spin-current in electron doped silicon can offer a promising path for spincaloritronics. Here, we create an electron spin polarization in the conduction band of n-type silicon by producing a temperature gradient across a ferromagnetic tunnel contact. The substrate heating experiments induce a large spin signal of $95 \mu \mathrm{V}$, corresponding to $0.54 \mathrm{meV}$ spin-splitting in the conduction band of $n$-type silicon by Seebeck spin tunneling mechanism. The thermal origin of the spin injection has been confirmed by the quadratic scaling of the spin signal with the Joule heating current and linear dependence with the heating power. (C) 2013 AIP Publishing LLC.

[http://dx.doi.org/10.1063/1.4845295]
\end{abstract}

Ultra large scale integration and miniaturization of electronic devices and circuitry yield to an increase in Joule heat dissipation and wastage of energy. An effort to reduce, reuse, or redirect this heat is not only of technological but also has fundamental and ecological importance. In this regard, caloritronics explores the possibility of directly converting heat into electricity. ${ }^{1}$ On the other hand, spintronics offers a promising path to achieve lower energy consumption. ${ }^{2,3}$ An exciting new research field taking advantage of both of these areas, called spin-caloritronics, is attracting a growing interest. ${ }^{4,5}$ Recently, many physical phenomena connecting spin to thermal effects have been proposed and demonstrated such as spin-Seebeck effect, ${ }^{6}$ spin dependent Seebeck effect in nanostructures and thermal spin injection into metal, ${ }^{7}$ thermal spin-transfer torque, ${ }^{8,9}$ spin-related Peltier cooling, ${ }^{10}$ and thermal spin tunnel effects. ${ }^{11,12}$ Especially interesting is the Seebeck spin tunneling into semiconductors, which is sensitive to the energy derivative of tunnel spin polarization (TSP) at the interfaces. ${ }^{12}$

The strong interest in Si for spintronics stems from its long spin coherence length, caused by the absence of hyperfine interactions and a weak spin-orbit coupling and its industrial dominance. ${ }^{13,14}$ Recently, electrical spin polarization in $\mathrm{Si}$ could be created at room temperature by spin polarized tunneling from a ferromagnet (FM). ${ }^{15-19}$ The Hanle and the inverse of the Hanle effects were used for the detection of spin polarization in different semiconductors under ferromagnetic tunnel contacts. ${ }^{20}$ Using such Hanle techniques, Le Breton et al. demonstrated a spin accumulation in hole doped p-type Si by using the Seebeck spin tunneling mechanism. ${ }^{12}$ In a FM/tunnel barrier/semiconductor structure, a temperature difference between the electrodes creates a tunnel spin current, ${ }^{12,21-23}$ which is governed by the energy derivative of the TSP. This involves thermal transfer of spin angular momentum from the ferromagnet to $\mathrm{Si}$ without net tunneling charge current. For Si based devices, these experiments are yet limited to reports on hole doped p-type $\mathrm{Si}$ with $\mathrm{Al}_{2} \mathrm{O}_{3} / \mathrm{NiFe}$ tunnel contacts. ${ }^{12}$ For further development, it is required to have spin tunnel contacts

\footnotetext{
a)andre.dankert@chalmers.se

b)saroj.dash@chalmers.se
}

with strong variation in TSP around the Fermi energy $\left(\mathrm{E}_{\mathrm{F}}\right)$, tunnel barriers with high interface thermal resistances, and its implementation in both electron and hole doped $\mathrm{Si}^{12}$ Therefore, a detailed investigation of the spin Seebeck tunneling effect with new ferromagnetic tunnel contact materials and electron doped n-type $\mathrm{Si}$ is crucial from fundamental and technological point of view.

In this Letter, we report a thermal creation of electron spin polarization in n-type Si by using the Seebeck spin tunneling mechanism. The temperature gradient between the n-type Si substrate and the FM yields to Seebeck spin tunnel currents, creating a large spin accumulation in the Si conduction band. We particularly use ferromagnetic tunnel contacts with ozone oxidized $\mathrm{SiO}_{2}$ tunnel barriers and $\mathrm{Co}$ ferromagnet on heavily doped n-type $\mathrm{Si}$. The detection of this thermally induced spin polarization was performed by employing Hanle and inverted Hanle effects in four-terminal measurement geometry. The amplitude of the thermal spin signal scales quadratically with the Joule heating current density and linearly with the heating power, which confirms the thermal origin of the observed Hanle signal. Electrical measurements and high magnetic field experiments rule out any other type of thermomagnetic effects. This result offers a way to generate a large electron spin accumulation in n-type Si by thermal spin injection using Seebeck spin tunneling.

For thermal spin injection into the n-type Si (measured electron density of $3 \times 10^{19} \mathrm{~cm}^{-3}$ at $300 \mathrm{~K}$ ), FM tunnel contacts $\left(\mathrm{Co} / \mathrm{SiO}_{2}\right)$ were fabricated on initially patterned $2 \mu \mathrm{m}$ thick silicon-on-insulator (SOI) channels (with the dimensions $200 \times 1000 \mu \mathrm{m}^{2}$ ). The $\mathrm{SiO}_{2}$ tunnel barrier was formed on the $\mathrm{Si}$ by ozone oxidation at room temperature. The chips were exposed to $\mathrm{O}_{3}$, created by a constant $\mathrm{O}_{2}$ flow $(1 \mathrm{l} / \mathrm{min})$ in the presence of a UV radiation source. This process results in uniform and pinhole-free tunnel barriers of $1.5 \mathrm{~nm}$ thickness. This is confirmed by the temperature and thickness dependent measurements of the junction resistance. ${ }^{29}$ Using such $\mathrm{SiO}_{2}$ tunnel barrier, a very large spin polarization in silicon could be achieved by electrical methods. ${ }^{29}$ After oxidation, the samples were transferred to an electron beam deposition system, where $15 \mathrm{~nm}$ Co and $10 \mathrm{~nm} \mathrm{Au}$ were deposited. The FM contacts were patterned by photolithography and Ar-ion beam etching. Subsequently, $\mathrm{Cr}(10 \mathrm{~nm}) / \mathrm{Au}$ 
$(100 \mathrm{~nm})$ contacts on the $\mathrm{Si}$ and contact pads on the ferromagnetic tunnel contacts were prepared by photolithography and lift-off technique. The $\mathrm{Cr} / \mathrm{Au}$ contacts were used for the Joule heating of the $\mathrm{Si}$ and as reference contacts to detect a voltage signal with respect to the FM tunnel contact. The ferromagnetic tunnel contacts have an area of $200 \times 100 \mu \mathrm{m}^{2}$ allowing a sufficiently good signal-to-noise ratio.

The principle for the thermal creation of spin accumulation in Si using the Seebeck spin tunneling mechanism is illustrated in Fig. 1. The simulated temperature distribution across the interface of the $\mathrm{Co} / \mathrm{SiO}_{2}(1.5 \mathrm{~nm}) / \mathrm{n}$-type Si device is shown in Figs. 1(a) and 1(b). The complete device structure of $\mathrm{Au}(100 \mathrm{~nm}) / \mathrm{Co}(15 \mathrm{~nm}) / \mathrm{SiO}_{2}(1.5 \mathrm{~nm}) / \mathrm{n}$-type $\mathrm{Si}$ $(2 \mu \mathrm{m})$ was considered for our calculation. A model was drawn to scale with our device and divided into finite elements using a commercial software (COMSOL) for calculating their temperatures. The temperature gradient $(\Delta T)$ is created by dissipation of an applied current density of $20 \mathrm{kA} / \mathrm{cm}^{2}$. The simulation takes Joule heating due to current flow and heat dissipation, transport, and radiation into account with a base temperature of $300 \mathrm{~K}$. Here, we have used a heat conductance $k^{S i O 2}=0.95 \mathrm{~W} \mathrm{~m}^{-1} \mathrm{~K}^{-1}$ for thin $\mathrm{SiO}_{2}$ tunnel barriers. ${ }^{26}$ Taking the large thermal conductivity of the Au pads and wire bonding into account, the surface temperature of the contact pads was fixed to $300 \mathrm{~K}$. This results in a temperature gradient $\Delta \mathrm{T}=500 \mathrm{mK}$ between the $\mathrm{Si}\left(\mathrm{T}_{\mathrm{Si}}\right)$ and $\mathrm{Co}\left(\mathrm{T}_{\mathrm{FM}}\right)$. This temperature difference yields to a nonequilibrium electron distributions in the Co and Si near the Fermi energy. This results in tunneling of equal number of electrons in opposite directions, giving rise to a zero net charge current. However, as the energy dependence of TSP is known to be different for the forward and reverse tunneling processes ${ }^{24,25}$ a spin accumulation $(\Delta \mu)$ can be induced in the Si (Fig. 1(c)). ${ }^{12,21}$

Before performing thermal spin injection experiments, we have tested the contacts for electrical spin injection and

(a)
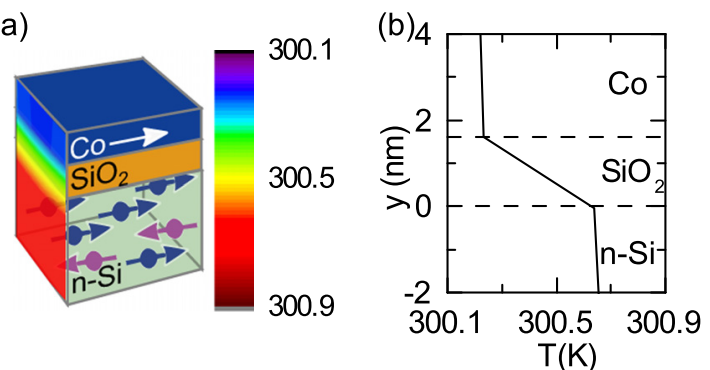

(c)

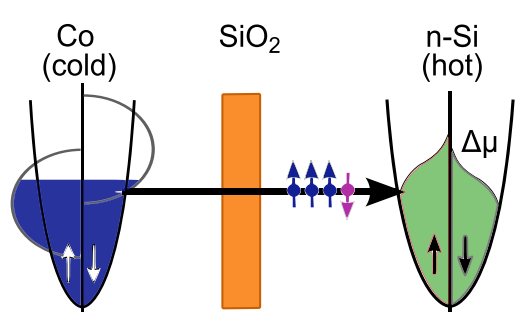

FIG. 1. (a) Device structure and simulated temperature distribution across the $\mathrm{Co} / \mathrm{SiO}_{2} /$ n-type $\mathrm{Si}$. (b) Temperature line scan across the tunnel contact for $1.5 \mathrm{~nm}$ thick $\mathrm{SiO}_{2}$ at heating current of $20 \mathrm{kA} / \mathrm{cm}^{2}$. (c) Spin-dependent density of states and its occupation for a tunnel contact with a hot Si and a cold Co electrode. The different energy dependence of tunnel spin polarization for forward and reverse tunneling process gives rise to a finite spin current, without any net charge current. verified the energy or bias dependence of the TSP. The measurements were performed in a three-terminal Hanle geometry as shown in Fig. 2(a). Electrical spin injection creates a majority spin accumulation and splitting of the electrochemical potential in the conduction band of the Si. The Hanle effect is used for the controlled reduction of the induced spin accumulation by applying a magnetic field $\left(\mathrm{B}_{\perp}\right)$ perpendicular to
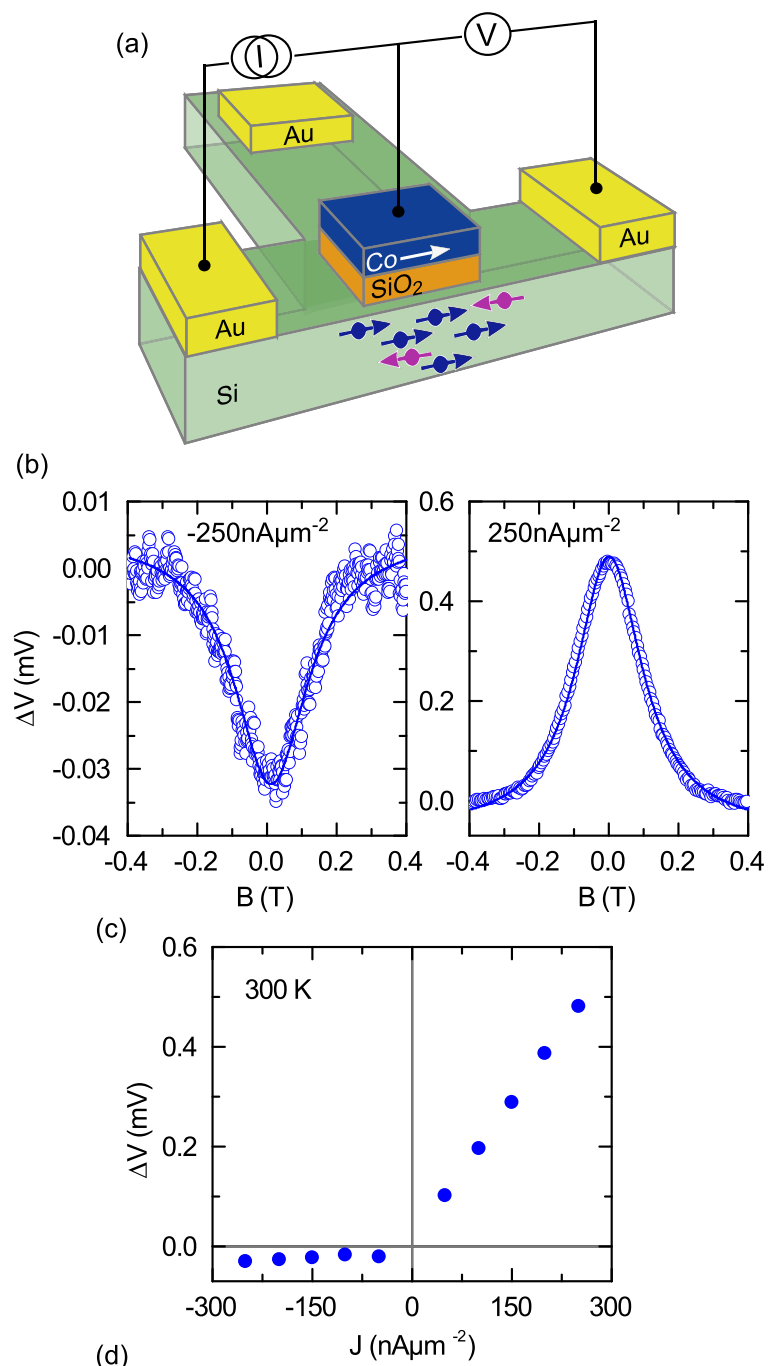

(d)

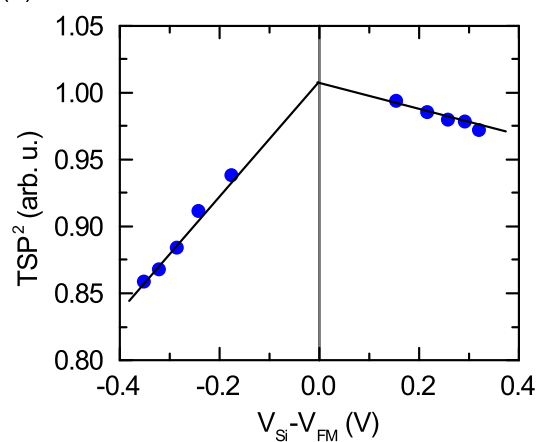

FIG. 2. Electrical Hanle effect at room temperature: (a) Three-terminal Hanle geometry for electrical injection and detection of spin polarization in the n-type $\mathrm{Si}$ with a $\mathrm{SiO}_{2}$-Co tunnel contact. (b) The electrical detection of spin polarizations in n-type $\mathrm{Si}$ at $300 \mathrm{~K}$ by Hanle effect for spin injection $\left(\mathrm{I}=+250 \mathrm{nA} \cdot \mu \mathrm{m}^{-2}\right)$ and spin extraction $\left(\mathrm{I}=-250 \mathrm{nA} \cdot \mu \mathrm{m}^{-2}\right)$. The solid lines are the Lorentzian fits with a lower limit for the spin life time $\tau_{s f}=50$ ps. (c) Electrical bias current dependence of the Hanle voltage signal $(\Delta \mathbf{V})$. (d) Electrical bias voltage dependence of $T S P^{2}$, which is proportional to the Spin-RA of the detected Hanle signal. 
the FM magnetization direction. The spin accumulation decays as a function of B with an approximately Lorentzian line shape given by $\Delta \mu\left(B_{\perp}\right)=\Delta \mu(0) /\left(1+\left(\omega_{L} \tau\right)^{2}\right)$, where $\Delta \mu(0)$ and $\Delta \mu\left(\mathrm{B}_{\perp}\right)$ are the spin accumulations in zero and finite perpendicular magnetic field, respectively, and $\tau_{s f}$ is the lower limit for spin lifetime. ${ }^{15,29}$ Figure 2(b) shows the measurement of the electrical Hanle signal for bias currents of $\pm 250 \mathrm{nA} \cdot \mu \mathrm{m}^{2}$ (corresponding bias voltage of $\pm 350 \mathrm{mV}$ ) at $300 \mathrm{~K}$. The spin injection $\left(\mathrm{V}_{\mathrm{Si}}-\mathrm{V}_{\mathrm{FM}}>0\right)$ from the $\mathrm{Co} / \mathrm{SiO}_{2}$ tunnel contact creates a majority spin $\left(\mathrm{n}^{\uparrow}\right)$ accumulation, whereas the spin extraction $\left(\mathrm{V}_{\mathrm{Si}^{-}}-\mathrm{V}_{\mathrm{FM}}<0\right)$ creates a minority spin $\left(n^{\downarrow}\right)$ accumulation in the Si. As expected, the measured Hanle signal for majority and minority spin accumulation are found to be of opposite sign. The spin accumulation $\Delta \mu$ scales with the TSP of the injected current and with spin life time $\left(\tau_{s f}\right)$. The detected voltage due to spin accumulation can be written as $\Delta V=T S P \times \Delta \mu / 2$. This results in the detected spin signal (spin resistance area product, spin RA) which is proportional to $T S P^{2} \times \tau_{s f}$. The variation of $T S P^{2}$ vs. bias voltage is presented in Fig. 2(d) and is found to be asymmetric. The strong decay of $T S P^{2}$ for energies above $E_{F}$ $\left(\mathrm{V}_{\mathrm{Si}}-\mathrm{V}_{\mathrm{FM}}<0\right)$ results in the direction dependent spin polarized tunnel currents. ${ }^{12,21}$ The difference in the spin polarization of the currents tunneling into and out of the $\mathrm{Si}$ can yield to a spin injection in our $\mathrm{Co} / \mathrm{SiO}_{2} / \mathrm{n}$-type $\mathrm{Si}$ devices. ${ }^{12,24,25}$ This asymmetry in the $T S P^{2}$ of $\mathrm{Co} / \mathrm{SiO}_{2} / \mathrm{n}$-type $\mathrm{Si}$ contact is essential to induce the large thermal spin accumulation in $\mathrm{Si}$ via the Seebeck spin tunneling mechanism.

The thermally induced electron spin accumulation in n-type $\mathrm{Si}$ has been created by introducing a temperature gradient between the $\mathrm{Si}\left(\mathrm{T}_{\mathrm{Si}}\right)$ and $\mathrm{Co}\left(\mathrm{T}_{\mathrm{FM}}\right)$ separated by a thin $\mathrm{SiO}_{2}$ tunnel barrier $(1.5 \mathrm{~nm})$. We increase the temperature of $\mathrm{Si}$ channel $\left(\mathrm{T}_{\mathrm{Si}}\right)$ by a DC current due to Joule heating. The $\mathrm{SiO}_{2}$ tunnel barrier prevents the current and heat from spreading into the ferromagnetic electrode. The temperature gradient created across the $\mathrm{SiO}_{2}$ barrier $\left(\mathrm{T}_{\mathrm{Si}}>\mathrm{T}_{\mathrm{FM}}\right)$ results in spin polarized Seebeck tunnelling through the oxide barrier, since the TSP for ingoing and outgoing currents differs. This creates a spin accumulation in the Si conduction band and therefore a splitting of the electrochemical potential $(\Delta \mu)$ as shown in Fig. 1(c). The detection of a thermally induced spin accumulation in $\mathrm{Si}$ is performed in both Hanle and inverted Hanle geometry using a $\mathrm{SiO}_{2} / \mathrm{Co}$ contact. ${ }^{12,15} \mathrm{~A}$ voltage signal is detected while sweeping a magnetic field, under the condition of zero net tunnel charge current $\left(\mathrm{I}_{\text {tunnel }}=0\right)$ between the $\mathrm{Co}$ and the $\mathrm{Si}^{12,15}$

A significant thermal Hanle spin accumulation signal of $\Delta \mathrm{V}_{\mathrm{H}}=50 \mu \mathrm{V}$ is observed in the Si for $\mathrm{T}_{\mathrm{Si}}>\mathrm{T}_{\mathrm{FM}}$, for a Joule heating current density of $\mathrm{J}= \pm 200 \mu \mathrm{A} / \mu \mathrm{m}^{2}$ (Fig. 3(b)). The Lorentzian line shape of the thermal Hanle signal obtained here is consistent with our electrical Hanle measurements. The magnitude and sign of the thermal Hanle curves are also identical for both directions of the Joule heating current. This is expected, since the Joule heating and therefore the gradient should be independent of the current direction. For $\mathrm{T}_{\mathrm{Si}}>\mathrm{T}_{\mathrm{FM}}$, a spin polarization identical to the electrical spin injection is observed, which corresponds to majority spin accumulation in the Si. This should be the case for a decaying TSP above $\mathrm{E}_{\mathrm{F}}$ as confirmed by our electrical bias (a)

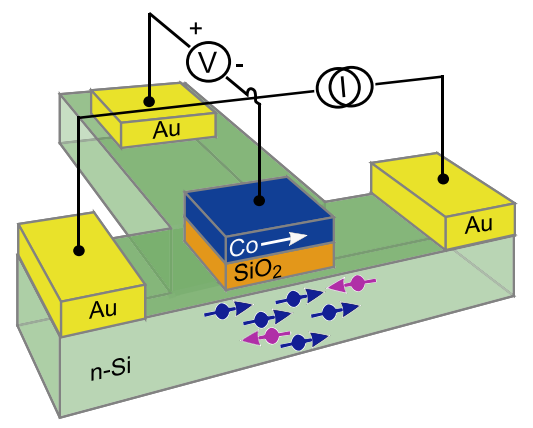

(b)

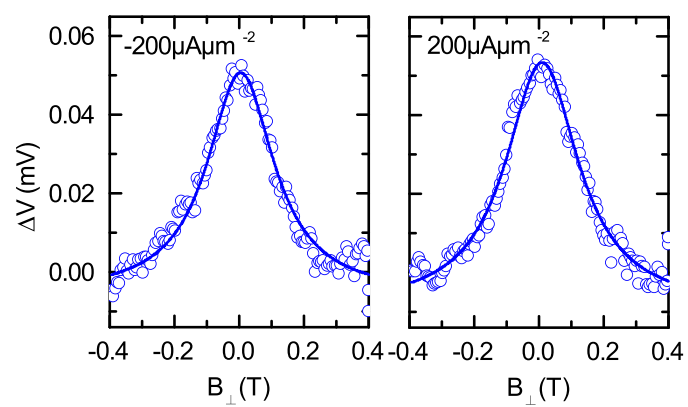

(c)

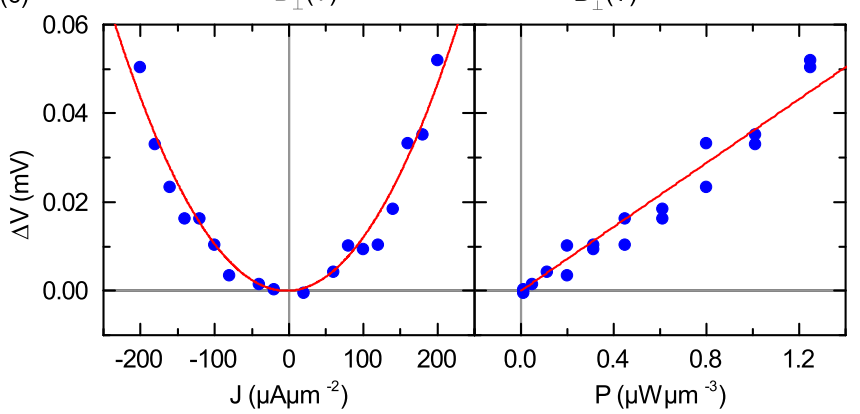

FIG. 3. Thermal Hanle effect at room temperature. (a) Device geometry and measurement scheme showing the Si Joule heating by a DC current, resulting in $\mathrm{T}_{\mathrm{Si}}>\mathrm{T}_{\mathrm{FM}}$. The voltage across the ferromagnetic tunnel contact is measured, with reference to another $\mathrm{Cr} / \mathrm{Au}$ contact placed on $\mathrm{Si}$. (b) Thermally induced spin accumulation in $\mathrm{Si}$, detected as a voltage change $(\Delta \mathrm{V})$ by the Hanle effect with perpendicular magnetic field. The plots show the data for heating current in two opposite directions $\left( \pm 200 \mu \mathrm{A} / \mu \mathrm{m}^{2}\right)$ at $300 \mathrm{~K}$. The solid lines are Lorentzian fits to the Hanle curves. (c) Left panel: Thermal Hanle signal $\Delta \mathrm{V}$ (symbols) versus Joule heating current density, with a quadratic fit (solid line). Right panel: $\Delta \mathrm{V}$ (symbols) as a function of Joule heating power and a linear fit (solid line).

dependence measurements presented in Fig. 2(d). Figure 3(c) shows the amplitude of the thermal Hanle signal measured for different Joule heating currents. The amplitude of the thermal spin signal is found to scale quadratically with the heating current density and hence linearly with the applied heating power, which confirms the thermal origin of the Hanle signal. ${ }^{12}$

Furthermore, we employ the inverted Hanle effect ${ }^{20}$ to detect the thermal creation of spin accumulations in $\mathrm{Si}$ at room temperature. The local magnetostatic fields arising from interface roughness in a ferromagnetic tunnel contact is known to reduce the spin accumulation in semiconductors. The inverted Hanle effect serves as experimental signature which recovers the reduced spin accumulation by applying an in-plane magnetic field $\mathrm{B}_{\|}{ }^{20}$ Figure 4 shows the measured data due to thermal spin injection for the case of $\mathrm{T}_{\mathrm{Si}}>$ $\mathrm{T}_{\mathrm{FM}}$ in the same $\mathrm{Co} / \mathrm{SiO}_{2} / \mathrm{n}$-type $\mathrm{Si}$ device. An inverted thermal Hanle signal of $\Delta \mathrm{V}_{\mathrm{iH}}=45 \mu \mathrm{V}$ could be detected for a current density of $\mathrm{J}= \pm 180 \mu \mathrm{A} \cdot \mu \mathrm{m}^{-2}$ (Fig. 4(a)). As the 
(a)

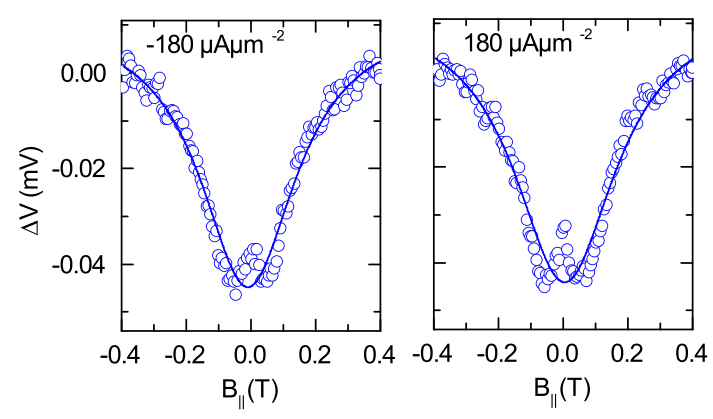

(b)

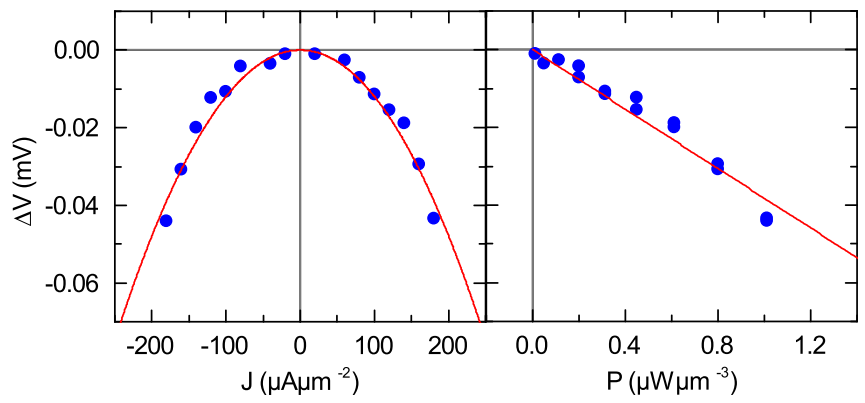

FIG. 4. Thermal inverted Hanle effect at room temperature. (a) Thermally induced spin accumulation in $\mathrm{Si}$, detected as a voltage change $(\Delta \mathrm{V})$ employing inverted Hanle effect with in-plane magnetic field. The plot shows the inverted Hanle data for heating currents of $\pm 180 \mu \mathrm{A} \cdot \mu \mathrm{m}^{-2}$ in two opposite directions. (b) Left panel: Thermal inverted-Hanle signal $\Delta \mathrm{V}$ (symbols) versus Joule heating current density, together with a quadratic fit (solid line). Right panel: $\Delta \mathrm{V}$ (symbols) as a function of Joule heating power and a linear fit (solid line).

Joule heating is independent of the current direction, spin signals of same sign and magnitude are obtained for both current directions. Figure 4(b) shows the amplitude of the inverted thermal Hanle signal for different Joule heating currents, which is also found to scale quadratically with the heating current density and linearly with the applied heating power. This scaling of the spin signal is consistent with a thermally induced spin accumulation in $\mathrm{Si}^{12}$

The total magnitude of the thermal spin accumulation in n-type $\mathrm{Si}$ is the sum of the Hanle $\left(\Delta \mathrm{V}_{\mathrm{H}}\right)$ and inverted Hanle $\left(\Delta \mathrm{V}_{\mathrm{iH}}\right)$ amplitudes. ${ }^{20} \mathrm{~A}$ total thermal spin signal of $\Delta \mathrm{V}=95 \mu \mathrm{V}$ corresponds to a spin splitting $\Delta \mu=2 \Delta V / T S P$ $=0.54 \mathrm{meV} \quad\left(T S P=0.35\right.$, assumed for $\mathrm{SiO}_{2} / \mathrm{Co}$ contact $)$. This corresponds to an electron spin polarization of $1 \%$ in the n-type Si conduction band created by thermal spin injection at room temperature, considering a parabolic conduction band and a Fermi-Dirac distributions for each spin direction ( $\mathrm{n}^{\uparrow}$ and $\mathrm{n}^{\downarrow}$ ). The magnitude of the thermal spin accumulation defined in terms of a Seebeck spin tunneling coefficient $\mathrm{S}_{\mathrm{st}}=\Delta \mu / \Delta \mathrm{T}$ is found to be $1.08 \mathrm{meV} / \mathrm{K}$. There can be some discrepancy with theory due to inaccuracy in the determination of $\Delta \mathrm{T}$, as experimental values for the thermal conductance of different materials and their interfaces are unknown. For the cases of thin tunnel barriers, the interface thermal resistance dominates over the bulk values and also differs with sample preparation conditions. In addition, the ballistic phonon transport and confinement effects in ultra-thin dielectrics and interface roughness play an important role in altering the thermal resistance of the contacts. ${ }^{26}$

The linear dependence of the spin signal on the heating power confirms the thermal origin of spin accumulation in n-

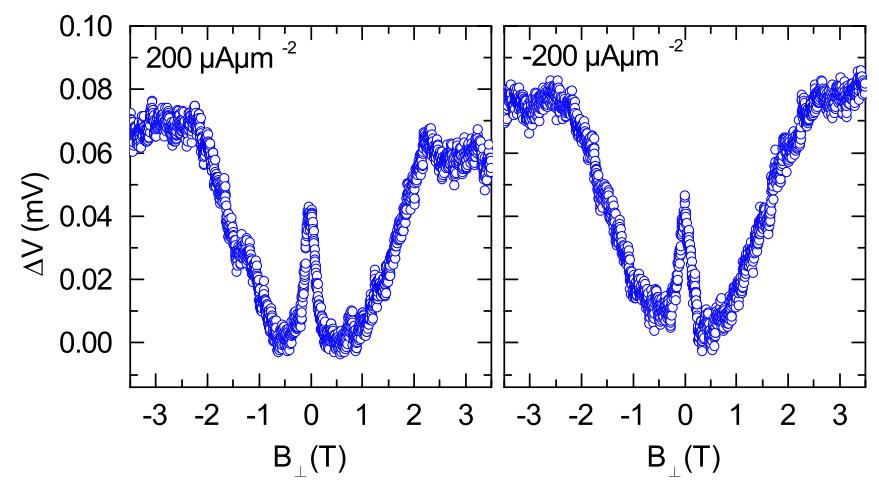

FIG. 5. Detection of thermally induced spin accumulation in Si with perpendicular field up to $3.5 \mathrm{~T}$, for Si heating currents of $\pm 200 \mu \mathrm{A} \cdot \mu \mathrm{m}^{-2}$. It shows the rotation and saturation of magnetization of ferromagnetic Co electrode to the out-of-plane direction at higher magnetic fields.

type Si. Furthermore, we can rule out other thermomagnetic effects such as spin related Hall, Nernst, and Ettingshausen effect, because they would not produce the characteristic Lorentzian shape Hanle signal. ${ }^{12}$ Nevertheless, to rule out all known thermomagnetic effects, measurements at high magnetic fields $\mathrm{B}_{\perp}$ were performed. For large enough $\mathrm{B}_{\perp}$, the magnetization of the FM rotates out of the plane. This aligns the injected spins parallel to the magnetization direction and produces zero precession, resulting in a recovery of the dephased spin signal. This recovery of the spin signal occurs only if the spin accumulation is produced by a transfer of the spins from the FM. The experimental data for the spin accumulation induced by heating of the $\mathrm{Si}$ electrode for $\mathrm{J}= \pm 200 \mu \mathrm{A} \cdot \mu \mathrm{m}^{-2}$ are shown in Fig. 5. Indeed, the recovery of the spin accumulation is observed when the magnetic field increases above $0.5 \mathrm{~T}$, with the signal saturating at about 2.5 T. This corresponds to the field at which the magnetization of the Co electrode has reached the full out-of-plane orientation. $^{27,28}$ The observation of the high-field recovery of the spin accumulation rules out the spin-Nernst effect and any other mechanism that does not involve transfer of spins from the FM to the $\mathrm{Si}^{12}$

In summary, we demonstrate the thermal creation of electron spin polarization in n-type Si using the Seebeck spin tunneling mechanism at room temperature. We show that the magnitude of thermally created electron spin polarization scales quadratic with the Joule heating current density and linearly with the heating power. A temperature gradient of few hundred milli-Kelvins across the ferromagnetic tunnel contacts creates a spin signal of more than $95 \mu \mathrm{V}$, which corresponds to a spin splitting of $0.54 \mathrm{meV}$ and spin polarization of $1 \%$ in the conduction band of n-type Si. Furthermore, the control experiment with thermal Hanle measurements at higher magnetic field and the comparison with electrical spin injection experiments rule out any other spurious effects. The advantage of utilizing the Seebeck spin tunneling mechanism is that the magnitude of spin accumulation is not limited to the TSP of ferromagnetic tunnel contact, but can be increased beyond that due to its dependence on energy derivative of TSP. Recently, similar results using $\mathrm{MgO}$ tunnel barrier have been reported, during the review process of our manuscript. ${ }^{30}$ By carefully designing the interfaces, Seebeck spin tunneling could be useful for creating spin polarizations 
in non-magnetic materials by itself or together with electrical spin injection and also for the reusing dissipated heat in electronic devices and circuits.

The authors acknowledge the support of colleagues at the Quantum Device Physics Laboratory and Nanofabrication Laboratory at Chalmers University of Technology. We would also like to acknowledge the financial supported from the Nano Area of the Advance program at Chalmers University of Technology, EU FP7 Marie Curie Career Integration grant and the Swedish Research Council (VR) Young Researchers Grant.

${ }^{1}$ G. Jeffrey Snyder and E. S. Toberer, Nature Mater. 7, 105-114 (2008).

${ }^{2}$ I. Zutić, J. Fabian, and S. Das Sarma, Rev. Mod. Phys. 76, 323 (2004).

${ }^{3}$ A. Fert, Rev. Mod. Phys. 80, 1517 (2008).

${ }^{4}$ G. E. W. Bauer, E. Saitoh, and B. J. van Wees, Nature Mater. 11, 391-399 (2012).

${ }^{5}$ M. Johnson and R. H. Silsbee, Phys. Rev. B 35, 4959 (1987).

${ }^{6}$ K. Uchida, S. Takahashi, K. Harii, J. Ieda, W. Koshibae, K. Ando, S. Maekawa, and E. Saitoh, Nature 455, 778-781 (2008).

${ }^{7}$ A. Slachter, F. L. Bakker, J. P. Adam, and B. J. van Wees, Nat. Phys. 6, 879-882 (2010).

${ }^{8}$ M. Hatami, G. E. W. Bauer, Q. Zhang, and P. J. Kelly, Phys. Rev. Lett. 99, 066603 (2007).

${ }^{9}$ H. Yu, S. Granville, D. P. Yu, and J.-Ph. Ansermet, Phys. Rev. Lett. 104, 146601 (2010).

${ }^{10}$ J. Flipse, F. L. Bakker, A. Slachter, F. K. Dejene, and B. J. van Wees, Nat. Nanotechnol. 7, 166-168 (2012).

${ }^{11}$ M. Walter, J. Walowski, V. Zbarsky, M. Münzenberg, M. Schäfers, D. Ebke, G. Reiss, A. Thomas, P. Peretzki, M. Seibt et al., Nature Mater. 10, 742-746 (2011).
${ }^{12}$ J. C. Le Breton, S. Sharma, H. Saito, S. Yuasa, and R. Jansen, Nature 475, 82 (2011).

${ }^{13}$ R. Jansen, Nature Mater. 11, 400 (2012).

${ }^{14}$ R. Jansen, S. P. Dash, S. Sharma, and B. C. Min, Semicond. Sci. Technol. 27, 083001 (2012).

${ }^{15}$ S. P. Dash, S. Sharma, R. S. Patel, M. P. de Jong, and R. Jansen, Nature 462, 491 (2009).

${ }^{16}$ R. Jansen, B. C. Min, S. P. Dash, S. Sharma, G. Kioseoglou, A. T. Hanbicki, O. M. J. van't Erve, P. E. Thompson, and B. T. Jonker, Phys. Rev. B 82, 241305 (2010).

${ }^{17}$ T. Sasaki, T. Oikawa, T. Suzuki, M. Shiraishi, Y. Suzuki, and K. Tagami, Appl. Phys. Express 2, 053003 (2009).

${ }^{18}$ K. R. Jeon, B. C. Min, I. J. Shin, C. Y. Park, H. S. Lee, Y. H. Jo, and S. C. Shin, Appl. Phys. Lett. 98, 262102 (2011).

${ }^{19}$ C. H. Li, O. M. J. van 't Erve, and B. T. Jonker, Nat. Commun. 2, 245 (2011).

${ }^{20}$ S. P. Dash, S. Sharma, J. C. Le Breton, J. Peiro, H. Jaffrès, J.-M. George, A. Lemaitre, and R. Jansen, Phys. Rev. B 84, 054410 (2011).

${ }^{21}$ R. Jansen, A. M. Deac, H. Saito, and S. Yuasa, Phys. Rev. B 85, 094401 (2012).

${ }^{22}$ A. Jain, C. Vergnaud, J. Peiro, J. C. Le Breton, E. Prestat, L. Louahadj, C. Portemont, C. Ducruet, V. Baltz, A. Marty et al., Appl. Phys. Lett. 101, 022402 (2012).

${ }^{23}$ K. R. Jeon, B. C. Min, S. Y. Park, K. D. Lee, H. S. Song, Y. H. Park, Y. H. Jo, and S. C. Shin, Sci. Rep. 2, 962 (2012).

${ }^{24}$ S. O. Valenzuela, D. J. Monsma, C. M. Marcus, V. Narayanamurti, and M. Tinkham, Phys. Rev. Lett. 94, 196601 (2005).

${ }^{25}$ B. G. Park, T. Banerjee, J. C. Lodder, and R. Jansen, Phys. Rev. Lett. 99, 217206 (2007).

${ }^{26}$ T. Yamane, N. Nagai, S. Katayama, and M. Todoki, J. Appl. Phys. 91, 9772 (2002).

${ }^{27}$ S. Sharma, S. P. Dash, H. Saito, S. Yuasa, B. J. van Wees, and R. Jansen, Phys. Rev. B 86, 165308 (2012).

${ }^{28}$ S. Sharma, A. Spiesser, S. P. Dash, S. Iba, S. Watanabe, B. J. van Wees, H. Saito, S. Yuasa, and R. Jansen, e-print arXiv:1211.4460.

${ }^{29}$ A. Dankert, R. S. Dulal, and S. P. Dash, Sci. Rep. 3, 3196 (2013).

${ }^{30}$ K. R. Jeon, B. C. Min, S. Y. Park, K. D. Lee, H. S. Song, Y. H. Park, and S. C. Shin, Appl. Phys. Lett. 103, 142401 (2013). 